\title{
REVERSIBLE CEREBRAL VASOCONSTRICTION SYNDROME IN PUERPERIUM: A PROSPECTIVE STUDY
}

ANZOLA Gian Paolo MD 1, BRIGHENTI Renato $\mathrm{MD}_{2}$, COBELLI Milena $\mathrm{MD}_{3}$, GIOSSI Alessia $\mathrm{MD}_{4}$, MAZZUCCO Sara MD5, OLIVATO Silvia MD 6 , PARI Elisa MD7, PIRAS Maria Paola $\mathrm{MD}_{8}$, PADOVANI Alessandro $\mathrm{MD}_{7}$, RINALDI Fabrizio $\mathrm{MD}_{7}$, TURRI Giulia MD 9

1. Consultant Neurologist -Villa Gemma Hospital - Gardone Riviera - Italy

2. Dept of Obstetrics and Gynaecology Poliambulanza Hospital - Brescia - Italy

3. Dept of Radiology Poliambulanza Hospital - Brescia - Italy

4. Dept of Neurology Azienda Socio Sanitaria Territoriale - Cremona - Italy

5. Nuffield Department of Clinical Neurosciences, University of Oxford, Oxford, UK.

6. ULSS 22 Verona - Italy

7. Dept of Neurology - Spedali Civili - University of Brescia - Brescia - Italy

8. Dept of Neurology Poliambulanza Hospital - Brescia - Italy

9. Department of Neurosciences, Biomedicine and Movement Sciences, University Hospital of Verona - Italy

Corresponding Author: Gian Paolo Anzola, MD

Consultant Neurologist

Casa di Cura Villa Gemma

Via Zanardelli 101 - 25083 Gardone Riviera (Brescia) - Italy

gpanzola@gmail.com 


\section{DISCLOSURES}

Dr Anzola received for this study a contribution from AFAR (Associazione Fatebenefratelli per la Ricerca)

Dr Brighenti reports no disclosure

Dr Cobelli reports no disclosure

Dr Giossi reports no disclosure

Dr Mazzucco reports no disclosure

Dr Olivato reports no disclosure

Dr Pari reports no disclosure

Dr Piras reports no disclosure

Dr Padovani reports no disclosure

Dr Rinaldi reports no disclosure

Dr Turri reports no disclosure

\section{CONTRIBUTIONS}

Dr Anzola conceived the study, did the statistical calculations and wrote the first draft of the paper.

Dr Brighenti organized the access to the patients

Dr Cobelli performed the neuroradiological investigations and provided material for the figures

Drs Giossi, Olivato, Pari, Piras, Rinaldi and Turri collected data

Dr Mazzucco supervised study researchers (Drs Olivato and Turri), critically revised the manuscript and gave important intellectual contributions

Dr Padovani supervised study researchers (Drs Giossi, Pari andRinaldi )

ABSTRACT WORD COUNT: 250

TEXT WORD COUNT: 2997

TITLE CHARACTERS COUNT: 76

TABLES: 1

FIGURES: 4

REFERENCES: 27

Running title: cerebral vasospasm during puerperium

Key words: cerebrovascular disease ,reversible cerebral vasoconstriction, thunderclap headache, puerperium 


\begin{abstract}
BACKGROUND AND AIM OF THE STUDY: Reversible cerebral vasoconstriction syndrome (RCVS) is characterized by severe "thunderclap" headache, with or without associated neurological symptoms and neuroimaging findings of reversible vasoconstriction of cerebral arteries.

Puerperium is a recognized precipitant, but the incidence of puerperal RCVS is unknown. We conducted a prospective study to assess incidence, risk factors and clinical features of RCVS.

MATERIAL AND METHOD: Nine-hundred consecutive puerperae were prospectively enrolled within three days of delivery. Past medical history, basal demographic, anthropometric and biological variables were recorded. Transcranial Colour Coded Sonography (TCCS) was performed to assess early signs of vasospasm in brain vessels. A structured telephone interview was planned in all subjects one month post delivery.
\end{abstract}

RESULTS: Thunderclap headache was recorded in 8 subjects $(0.9 \%)$ on the first visit. At the one month follow-up interview 27 more patients reported having had at least one episode of thunderclap headache. In these $33(3.8 \%)$ patients the course was spontaneously benign. .

One patient presented to the Emergency ward with throbbing thunderclap headache three weeks after delivery. Diagnostic work-up ended up in the diagnosis of RCVS, the outcome was favorable CONCLUSION: In normally coursing pregnancies and after uncomplicated delivery the risk of puerperal RCVS is negligible $(0.1 \%)$. On the other way thunderclap headache may occur in a measurable proportion of $(3.4 \%)$, although in the vast majority of cases $(33 / 34=97 \%)$ it is of benign course. Transcranial Doppler sonography may be helpful to pick up those cases in whom further neuroradiological investigation is warranted. 


\section{INTRODUCTION}

Reversible cerebral vasoconstriction syndrome (RCVS) is a unified term used to describe a group of disorders sharing definite angiographic and clinical features. It is clinically characterized by severe headache, with or without other acute neurological symptoms (such as seizures, hemiparesis or encephalopathy) with neuroimaging findings of reversible segmental and multifocal vasoconstriction of cerebral arteries. These clinico-radiological syndromes are usually self-limited to 3 months $(1,2,3,4,5)$.

Usually the first symptom is a thunderclap headache, described as a severe pain peaking in less than one minute, often accompanied by nausea, less often vomiting, photophobia, blurred vision and transient motor deficits. Typically the head pain recurs intermittently for $1-2$ weeks $(2,6,7)$. The major complications of the syndrome are ischemic and hemorrhagic strokes which may bring about severe disability .

Incidence is unknown, but it is considered a rare clinical entity, reaching $0.26 \%$ of patients presenting to an emergency headache clinic (8). A middle aged female preponderance is reported (9). Approximately $60 \%$ of cases are related to specific risk factors, including vasoactive drugs, cervical and cerebral large-artery lesions, neurosurgical procedures, endocrine abnormalities and post-partum period (10). Recent literature data show that $7-9 \%$ of all RCVS cases occurred in the post-partum setting $(2,8,4)$.

Post-partum RCVS (PPRCVS) usually starts within one month from delivery, in 2/3 of cases during the first week $(5,9)$. Therefore it is to be considered among possible etiologies in the broader spectra of both post-partum angiopathy and post-partum headache, both of which are particularly frequent in the first post delivery month $(11,12,13,14,15,16,17)$.

PPRCVS is deemed to entail a favourable prognosis, although in a recent review focal deficits were reported in 75 out of 98 cases (9) and fulminant cases have been observed $(18,19)$. The retrospective nature of all reported cases, however, makes both the true outlook and true incidence unclear and it is possible that PPRCVS is largely underestimated, like RCVS occurring outside puerperium (5)

We therefore conducted a prospective study in consecutive women giving birth to a child to assess incidence, risk factors and clinical features of PPRCVS. 


\section{MATERIAL AND METHODS}

The study period extends from November 2011 to July 2013. We studied all female subjects having given birth to a baby in the Obstetrics Department of the S. Orsola Hospital (first 86 cases, then Poliambulanza Hospital, as the former was incorporated in the latter ) in Brescia, Italy. The subjects underwent a basal visit within $96 \mathrm{hr}$ of delivery, in which a chart review was performed and a structured interview was administered in person by one research assistant, with particular emphasis on headache. Thereafter, ABP was measured noninvasively and a transcranial ultrasound study was performed.

The study protocol was approved by the S. Orsola Hospital Institutional Review Board. All patients provided informed consent before entering the study.

Information was collected on maternal demographics, labour and delivery details, anaesthesia during labour and delivery and headache characteristics and management. The following data were also collected: age, ethnicity, height, weight gain, arterial blood-pressure, haemoglobin, uric acid and proteinuria level, history of primary headache disorders, history of arterial hypertension, smoking habits, alcohol intake, diabetes, dyslipidemia, family history of stroke and detailed characteristics of recent pregnancy and delivery, including medications and drugs taken during pregnancy.

Headache intensity was evaluated by using a visual analog scale ranging from 0 (no pain) to 10 (the maximum pain that you can ever imagine). Headache attacks reaching a maximum intensity above $7 / 10$ in less than 1 min were qualified as 'thunderclap headache' whereas those peaking more slowly as 'acute severe headache'. Headache diagnosis was made according to the 2004 criteria of the International Headache Society (1). Thunderclap headache was used as sentinel symptom to raise suspicion of possible PPRCVS

A basal assessment with Transcranial Colour-Coded Sonography (TCCS) was performed in all patients to detect any early sign of vasospasm with a Philips IU 21 device. The M1 and M2 segments of the middle cerebral artery (MCA) were identified on colour representation and mean flow velocities (MFV) were recorded (20). Submandibular windows were used to measure flow velocity in the distal internal carotid artery (ICA). MFV of $100 \mathrm{~cm} / \mathrm{sec}$ was selected as the normal upper limit in this study, according to threshold criteria derived from the literature (21); any higher value was considered suggestive of MCA stenosis. In patients with MCA velocity exceeding the threshold, we also calculated Lindegaard Index (LI) by dividing the MFV in MCA by the MFV of ipsilateral distal extracranial ICA . Published studies have indicated that an LI of 3 to 6 can be 
considered as mild vasospasm and greater than 6 can be considered moderate-to severe vasospasm (22).

All subjects were further instructed to pay special attention to the occurrence of sudden thunderclap headache in the following month and in case to access the Emergency Ward of the Hospital for expedited assessment or to contact by phone one of the study researchers. All patients presenting with thunderclap headache underwent a detailed neurological examination and a repeat transcranial Doppler. If TCCS showed signs of MCA vasospasm the patient performed a brain MRI, including angiography (MRA) on a 1.5 Tesla MRI machine.

To ensure complete ascertainment all the participants were reassessed at one month after hospital discharge by a structured telephone interview inquiring about the occurrence and detailed characteristics of any type of headache, focal neurological deficits and seizures since delivery. Continuous variables were compared by mean of two-tailed $\mathrm{T}$ test if normally distributed or non parametric tests if the distribution was not normal. Frequencies were compared by Chi square test (SPSS version 22). 


\section{RESULTS}

During the inclusion period of 20 months, 900 women were enrolled in the study: $75 \%$ of them were of Caucasian ethnicity, mean age was $31 \pm 5$ (range 16-45), 53\% at their first pregnancy, 32\% at the second one, the remaining $15 \%$ being distributed to up to six previous pregnancies. They were evaluated at a mean of $1.5 \pm 1$ days post partum (range $0-11$ ), and follow-up telephone interview occurred on the average $39 \pm 11$ days (range 25-50) after childbirth. Two-hundredninety-two $(32.2 \%)$ were migraine sufferers and 164 (19.3\%) reported tension type headache in past history.

Thunderclap headache was recorded in 8 subjects $(0.9 \%)$ on the first visit

On basal TCCS assessment, flow velocities were overall symmetrically and evenly distributed and in the upper range of normal values ( right M1 and M2 $74 \pm 17$ and $72 \pm 17 \mathrm{~cm} / \mathrm{sec}$ respectively; left M1 and left M2 73 17 and 72 $\pm 17 \mathrm{~cm} / \mathrm{sec}$ respectively). One-hundred thirty-six subjects $(12.1 \%)$ exceeded in at least 1 measurement the threshold of $100 \mathrm{~cm} / \mathrm{sec}$, but LI was consistently $<3$ in all of them, thus suggesting that velocity increase was not caused by intracranial vasospasm. Comparing subjects with MFV $\geq 100 \mathrm{~cm} / \mathrm{sec}$ with those below for anthropometric and biological variables, $\mathrm{Hb}$ was significantly lower in patients with $\mathrm{MFV} \geq 100 \mathrm{~cm} / \mathrm{sec}(10.8 \pm 0.2$ vs. $11.5 \pm 0.1$ $\mathrm{g} / \mathrm{lt}$ respectively $-\mathrm{p}<.0001$ on t-test). Since MFV as measured by transcranial Doppler is known to be negatively correlated with haematocrit (23) the most obvious explanation for the raised velocities is the blood loss related to delivery.

None of the 8 subject who reported having suffered from a thunderclap headache soon after delivery had neurological abnormalities nor ultrasound findings suggestive of vasospasm.

During the follow-up period six women contacted the research staff for the occurrence of headache with hyper acute onset. They were all examined and subjected to MRA: both clinical, neurosonological and neuroradiological findings were within normal limits. On final follow-up interview, 241 subjects (26.8\%) reported having had at least one headache attack since delivery, in 27 of them with features of thunderclap headache. None of the 27 women with thunderclap headache ever accessed Emergency Ward or sought medical advice, all were free of headache at the time of follow-up and none reported focal deficits. Therefore, overall 33 women (3.8\%) had at least one attack of thunderclap headache at the end of follow-up None of the variables assessed at baseline, including history of migraine, differentiated patients with from those without thunderclap headache on follow-up. (table 1). 


\begin{tabular}{|l|c|c|c|}
\hline & $\begin{array}{c}\text { Thunderclap } \\
\text { headache } \\
\mathrm{n} .=34\end{array}$ & $\begin{array}{c}\text { No headache } \\
\mathrm{n} .=659\end{array}$ \\
\hline BMI & $23 \pm 4$ & $23 \pm 4$ & NS \\
\hline AGE & $30 \pm 6$ & $31 \pm 5$ & NS \\
\hline Weight gain in Kg & $12 \pm 4$ & $13 \pm 5$ & NS \\
\hline RIGHT M1 MFV & $75 \pm 15$ & $74 \pm 17$ & NS \\
\hline RIGHT M2 MFV & $75 \pm 20$ & $72 \pm 17$ & NS \\
\hline LEFT M1 MFV & $78 \pm 17$ & $73 \pm 17$ & NS \\
\hline LEFT M2 MFV & $76 \pm 18$ & $72 \pm 17$ & NS \\
\hline SYSTOLIC BP & $112 \pm 12$ & $111 \pm 12$ & NS \\
\hline DIASTOLIC BP & $70 \pm 8$ & $68 \pm 9$ & NS \\
\hline Hb & $11,3 \pm 1$ & $11,3 \pm 1,3$ & $30,3 \%$ \\
\hline History of migraine & $29,9 \%$ & & NS \\
\hline
\end{tabular}

TABLE 1: comparison between subjects who reported the occurrence of thunderclap headache and women without headache for potentially meaningful variables assessed at baseline.

One patient (0.1\%) presented to the Emergency Ward for acute onset of throbbing "worst in the lifetime" headache. Clinical details are given in the following section.

\section{CASE REPORT}

On December 2nd 2011 A.A.B., a 31 year old black woman, gave birth to a healthy child through caesarean delivery with spinal anaesthesia after an uncomplicated pregnancy of 37 weeks.

Personal and family history were unremarkable, except for $10 \mathrm{Kg}$ weight gain (BMI 27,3). She reported no history of headache, no diabetes, no hypertension, no use of illicit drugs. She hadn't taken any medication during pregnancy. Her previous pregnancy had been unremarkable and she had given birth to a healthy baby without complications.

She was examined three day after delivery and did not report headache. General and neurological examinations were normal, ABP 120/70 Hg mm. TCCS showed normal flow velocities on both 
MCAs. On December $6^{\text {th }} 2012$ she was discharged. The day after, she developed sudden, throbbing and severe headache, lasting 24 hours, located in the right side of the head, without preceding aura, nausea or vomiting, only partially relieved by acetaminophen, which recurred daily over the next 20 days

On clinical examination, December $27^{\text {th }}$, she appeared fully alert and cooperative. No focal deficits were noticed. Her ABP was 115/80 Hg mm. Throbbing headache was still present and distressing (intensity 8/10). TCCS showed raised MFV on distal left MCA $(117 \mathrm{~cm} / \mathrm{sec})$, whereas the other findings were within normal limits ( Left M1 $88 \mathrm{~cm} / \mathrm{sec}$, right M1 $83 \mathrm{~cm} / \mathrm{sec}$, right M2 $85 \mathrm{~cm} / \mathrm{sec}$ ). She was hospitalized. Urgent MRI, performed on December $30^{\text {th }}$ with a 1.5 tesla GE Signa Excite HDXT, showed multiple small foci of hyperintensity on T2 FLAIR-weighted imaging with normal findings on diffusion-weighted imaging located in the frontal and posterior temporal cortices bilaterally (see figures 1 and 2). MRA showed mild stenosis of the left carotid siphon and of distal right intracranial vertebral artery (see figure 3).

Blood and urine routine tests were normal. In the following two days headache resolved after i.v. ketoprofen $100 \mathrm{mg}$ daily. No further headache was recorded during hospital stay. On the tenth day after hospitalization TCCS exhibited normal values on both sides and the patient was discharged. Repeat MRI/MRA, performed one week after discharge, showed the disappearance of the parenchymal abnormalities and normalization of vessel diameter (see figure 1, 2 and 3).

Furthermore left posterior communicating artery appeared significantly wider compared with the first examination, suggesting resolution of previous spasm (figure 4).

On telephone follow up three months later the patient was still asymptomatic and free of headache. 

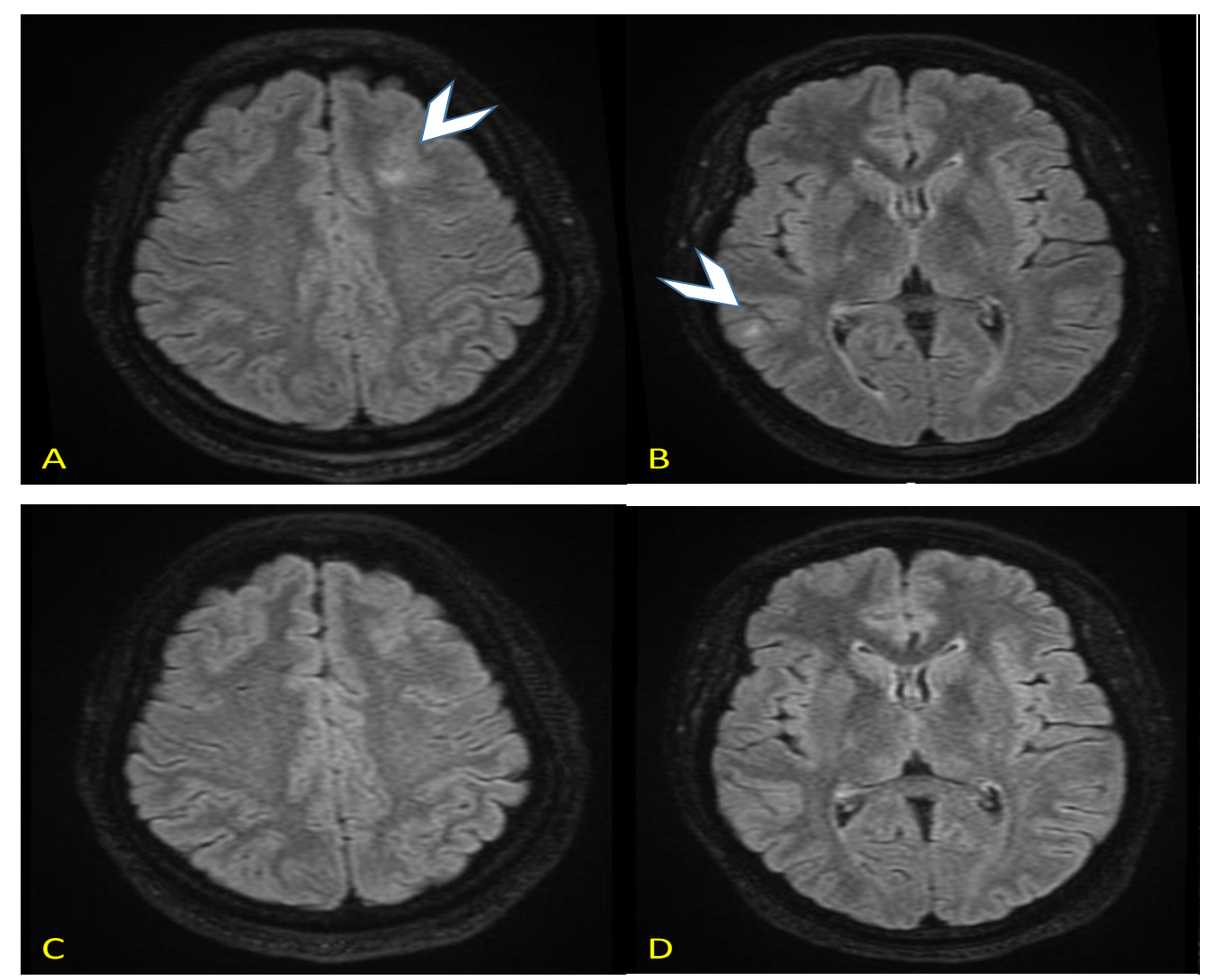

Figure 1: T2 FLAIR-weighted MRI, axial view

Upper panel: Small cortical hyper intense foci in the left frontal (arrowhead A) and right posterior temporal cortex (arrowhead B) .

Lower panel: Complete resolution of the abnormalities on repeat examination. 

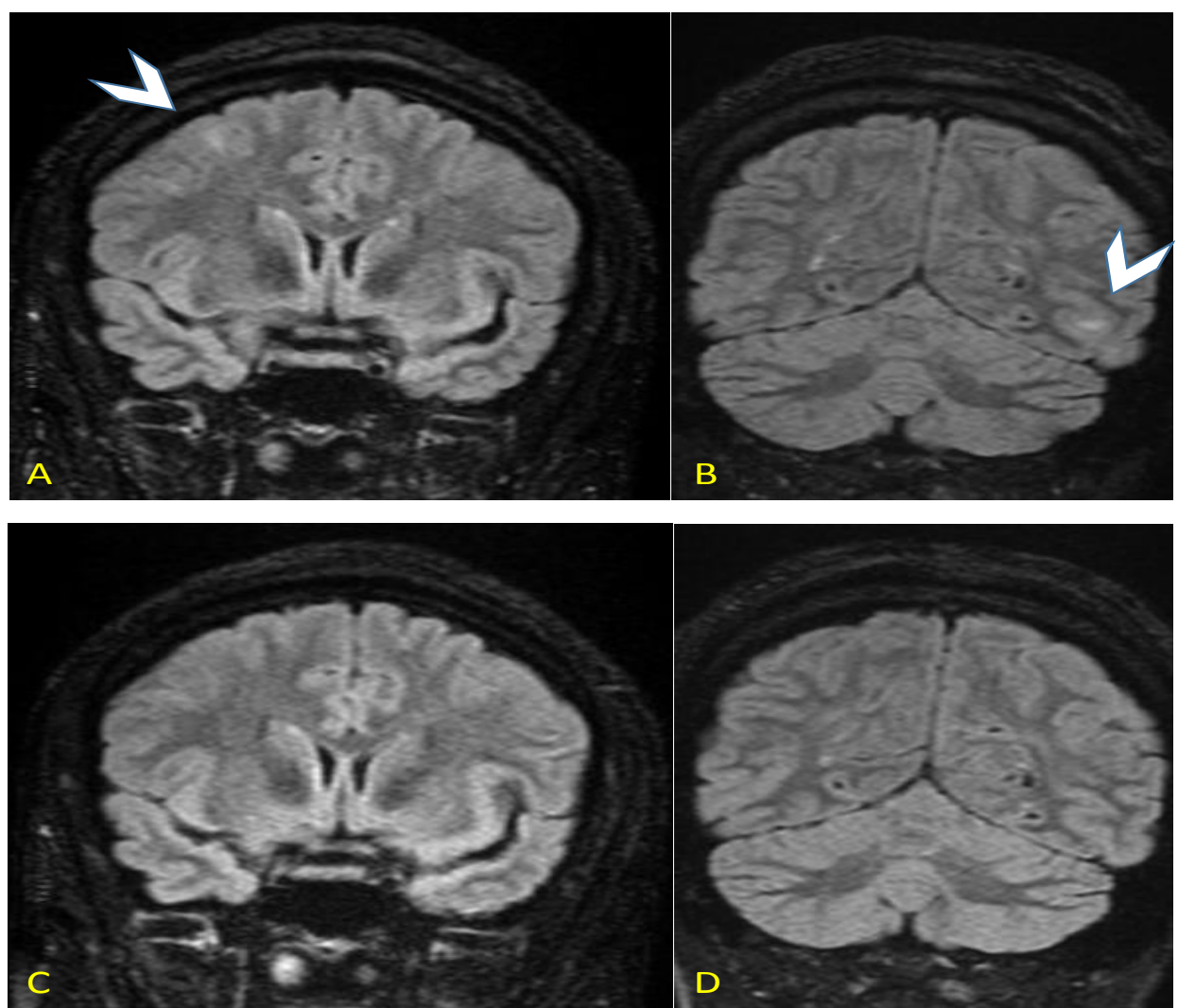

Figure 2: T2 FLAIR-weighted MRI, coronal view

Upper panel: Small cortical hyper intense foci in the right frontal (arrowhead A) and left posterior (arrowhead B) temporal cortex.

Lower panel: Complete resolution of the abnormalities on repeat examination. 

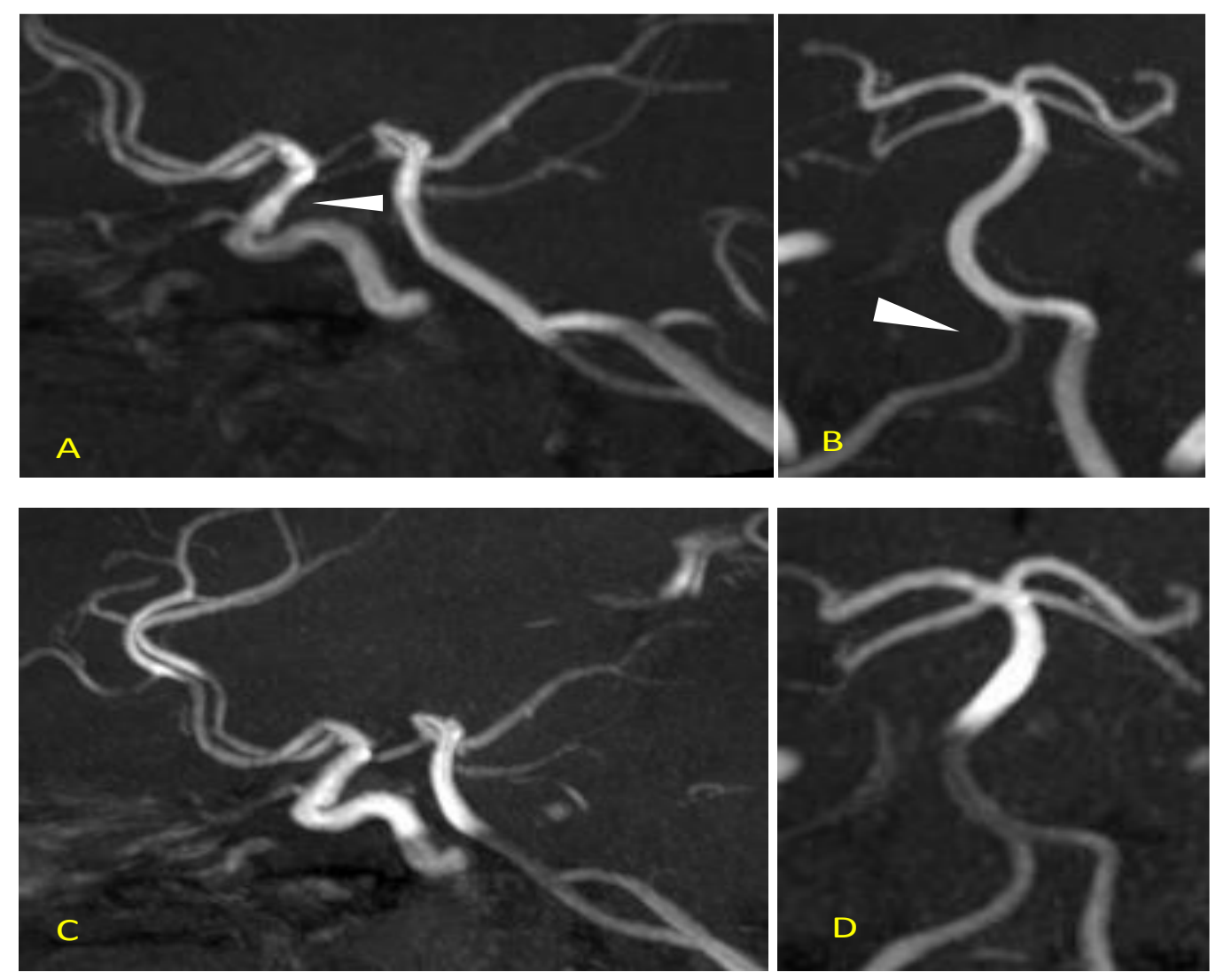

Figure 3: MRA :

Upper panel: mild stenosis of left siphon (arrowhead A) and left distal vertebral artery (arrowhead B).

Lower panel: resolution of stenosis in the left siphon (C) and left distal vertebral artery (D). 

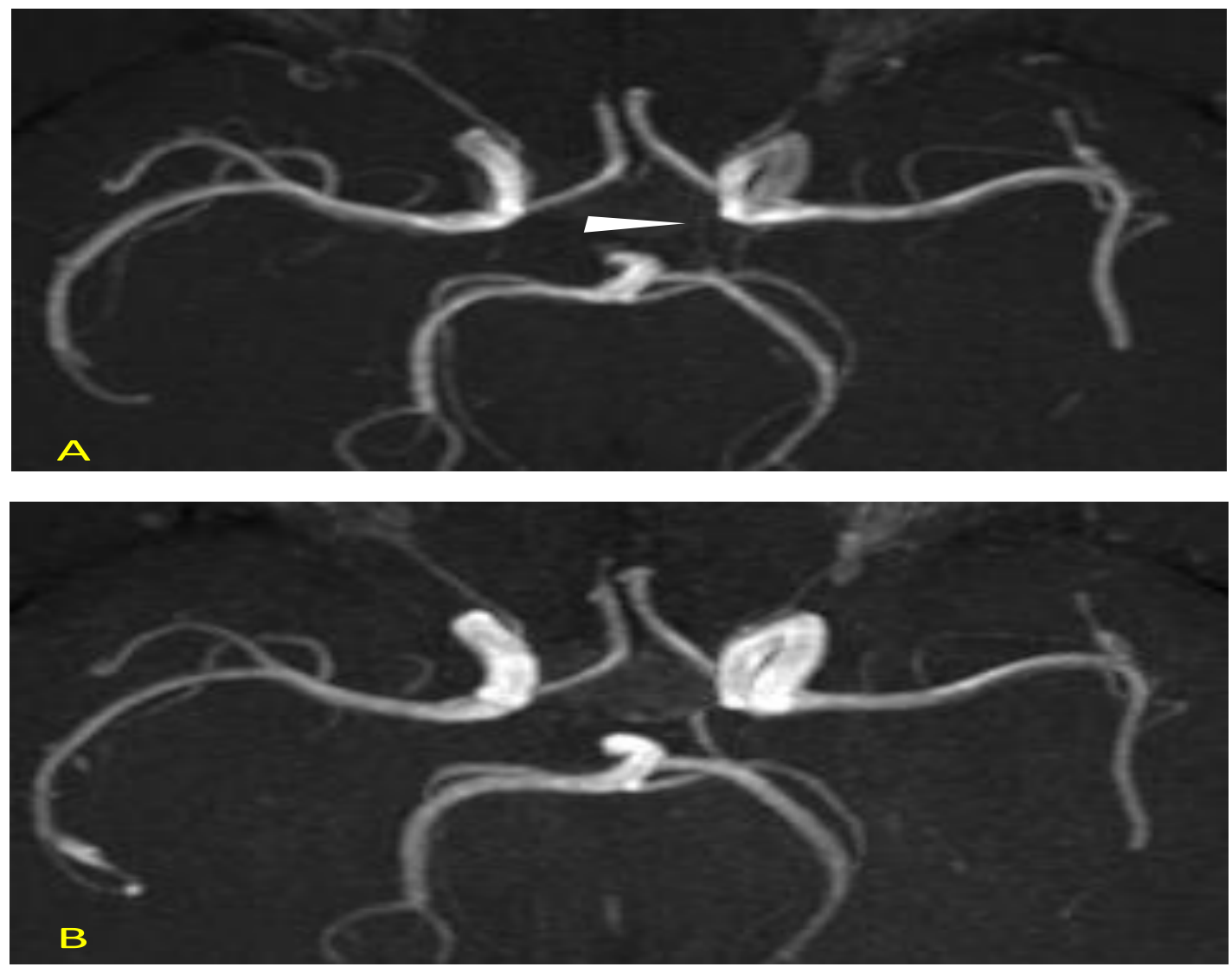

Figure 4: MRA

Upper panel: Right posterior communicating artery is barely visible (arrowhead - A).

Lower panel: Right posterior communicating artery is now fully visualized (B). 


\section{DISCUSSION}

Reversible vasoconstriction syndrome is a relatively recently recognized disease of brain vasculature the cardinal signs of which are abrupt headache with "thunderclap" features, most of the times in recurring attacks, and segmental vasoconstriction of brain vessels with variable neuroradiological abnormalities, including subarachnoid hemorrhage, and mainly favourable outcome $(5,7,24)$.

Puerperium has been identified as a trigger factor mainly because about $10 \%$ of the reported cases occurred within one month of delivery (9).

PPRCVS is probably a benign disorder, but fulminant cases have been reported $(9,18,19)$, which highlights the need of an early diagnosis.

The present study was undertaken to assess the incidence of PPRCVS in a prospective way and to look for possible predisposing factors. We chose to highlight the occurrence of thunderclap headache as hallmark to raise suspicion of PPRCVS and TCCS as a screening and follow-up method. Thunderclap headache is invariably reported as the presenting symptom: in a recent review Skeik et al found severe headache in 95/98 patients with PPRCVS and overall in RCVS headache has been reported as the presenting complaint in $95 \%$ to $100 \%$ of cases $(4,5)$.

Transcranial sonography was successfully employed by Chen and associates to monitor vasospasm in 32 patients with proven RCVS, with a threshold value of $80 \mathrm{~cm} / \mathrm{sec}$ as upper limit of normal (26). We had to reset the criterion for vasospasm, as a significant number of subjects had MFV values exceeding $100 \mathrm{~cm} / \mathrm{sec}$ at baseline evaluation due to an anaemic state. Therefore, we dismissed absolute velocities in favour of the Lindegaard index as a screening measure. In fact, increased blood flow velocities in the middle cerebral artery may either result from vasospasm of MCA main trunk or from overall increased blood flow in the MCA territory. In the former case the acceleration is limited to intracranial vessel, whereas in the latter it involves also distal ICA. Therefore, the MCA/ICA ratio of blood flow velocity (The so called Lindegaard index) is elevated in cases of intracranial vasoconstriction but remains in the normal limit of $<3$ when blood flow velocity is increased to the same extent in MCA and ICA.

Thunderclap headache was by no means a rarity in our cohort, as it was already noticed in about $1 \%$ of women in the first three day post partum. In no case, however, was TCCS suggestive of vasospasm at the basal evaluation. During the first month post delivery, thunderclap headache occurred in $3.8 \%$ of all included women, with a benign course. In six of these cases, who sought 
medical advice, clinical, neurosonological and neuroradiological findings were negative for PPRCVS. The remaining cases were identified on the final follow-up interview. We did not perform a repeat TCCS in these subjects. However, basal ultrasound assessment was normal in all, symptoms had resolved spontaneously at the time of follow-up interview, none had sought medical advice at the time of headache occurrence and subjects were asymptomatic at the moment of the follow-up assessment. Therefore we deemed any further investigation unnecessary. The six examined patients were also interviewed at the end of the follow-up period. All fared well and reported resolution of symptoms. The occurrence of single episodes of thunderclap headache in the 27 subjects who reported it on the final interview occurred haphazard in the first month post delivery. At the time of the interview all these women reported well being. Therefore TCCS was not deemed necessary. It is possible that some of these cases represent minor episodes of subtle vasospasm. However, none of the 6 additional patients who were examined during follow up showed any abnormality in brain vessels. For these reason the probability of pathological findings is very low in our opinion.

In only one case, the occurrence, intensity and duration of headache brought the patient to the Emergency Ward : the clinical picture and instrumental findings fulfilled the criteria for RCVS proposed by Ducros in 2012 (5), except for the lack of CSF examination. This was reputed unnecessary given the very benign clinical outcome under non steroidal anti inflammatory agents and the absence of visible aneurysms on MRA. Primary angiitis was likewise excluded on the basis of the combination of clinical (headache type) and MRA findings.

MRI showed features of posterior reversible encephalopathy (PRES), i.e. patchy areas of vasogenic oedema which resolved in 7 days (see figures 1 and 2). Of notice is the fact that the distribution of lesions, at the cortico-subcortical junction in scattered foci, point to the involvement of pial arteries, suggesting that the pathological process involved not only main arterial trunks, as assessed by MRA, but also more distal terminal vessels (5).

PRES has been reported independently of RCVS (27), but it is likely that the two conditions overlap to some extent, because as many as $30 \%$ of documented RCVS cases in the literature show features of PRES on MRI $(4,26)$.

Compared with the literature the onset of symptoms was in the time range of most reported cases (5-7 days after delivery), duration of symptoms was likewise of about three weeks but the clinical picture was very benign, being limited to severe headache. Nevertheless, the neuroradiological picture showed both vasoconstriction in at least two segments (although not severe) and parenchymal abnormalities, and TCCS a focal velocity increase. Parenchymal abnormalities were 
located in the territory of pial arteries, which are not visualized by MRA, whereas TCCS suggested vasospasm in the distal M2 segment of left MCA, the latter not being confirmed by MRA ( see figure 3). However, MRA was performed some day after TCCS. Narrowing of vessels is probably a dynamic process in RCVS, which has been suggested to follow a backward course from pial to main basal trunk $(5,24,26)$. Therefore we speculate that distal left MCA stenosis was no more visible on MRA because it had already resolved at the time of the neuroradiological investigation.

The short and benign course in our case points to the possibility that many more cases of PPRCVS may pass undetected because of the spontaneous remission of symptoms and possibly also of the abnormalities on imaging.

No known trigger factor was identifiable in the present case, except perhaps spinal anaesthesia (5, 9). However, spinal anaesthesia does not appear to be a very powerful trigger in itself, as the PPRCVS case was the only one among 131 spinal anaesthesias recorded in our series $(0.7 \%$ - data not shown).

In conclusion: in our prospective series of women having given birth to a baby the systematic search of signs and symptoms of PPRCVS showed an incidence of $0,11 \%$ (95\% C .I. $=0.103-$ 0.117), with only one very mild case out of 900 women investigated. We acknowledge the lack of investigation in 33 cases who had had at least one episode of acute onset headache, but for the reasons enumerated above we don't think we missed any significant case of PPRCVS. In any case, in the worst scenario of 34/900 cases, incidence would have been $3 \%$ of however clinically irrelevant cases with $95 \%$ C.I. ranging from $1,89 \%$ to $4.1 \%$. It is thus likely that the incidence of clinically meaningful cases is even lower and the emphasis given to the puerperium as a trigger factor has been overestimated due to a selection bias.

Our data suggest that in normally coursing pregnancies and after uncomplicated delivery ( be it vaginal or caesarean) the risk of PPRCVS is negligible.

On the other way thunderclap headache, which is the hallmark of PPRCVS, may occur in a proportion of women in the first post partum month, although in the vast majority of cases ( $33 / 34=$ $97 \%$ ) it is of benign course and will resolve spontaneously with no need for neuroradiological investigations. It can be questioned whether these cases represent examples of truly "primary" thunderclap headache (28) or mild PPRCVS cases.

Transcranial Doppler may be helpful in identifying associated vasospasm by showing focal increases in MFV, but there is need for better redefinition of velocity thresholds because in the first post partum days or weeks intracranial flow velocities may be high for reasons other than focal 
vasoconstriction. Pending validation studies, the use of Lindegaard Index is recommended instead of velocimetry to pick up truly positive cases.

Merits of the present study are the prospective nature, the large cohort size and the systematic investigation of all included cases and follow up with no lost, which allowed complete case ascertainment.

The main limit of the study was that it was based on a single centre experience. 


\section{REFERENCES}

1. Headache classification subcommittee of the International Headache Society. The international classification of headache disorders. Cephalalgia 2004; 24: 1-160.

2. Ducros A, Boukobza M, Porcher R, Sarov M, Valade D, Bousser MG. The clinical and radiological spectrum of reversible cerebral vasoconstriction syndrome: a prospective series of 67 patients. Brain 2007; 130: 3091-101.

3. Calabrese LH, Dodick DW, Schwedt TJ, Singhal A. Narrative Review: Reversible Cerebral Vasocontriction Syndromes. Ann Intern Med 2007; 146: 34-44.

4. Singhal AB, Hajj-Ali RA, Topcuoglu MA, et al. Reversible cerebral vasoconstriction syndromes: analysis of 139 cases. Arch Neurol 2011; 68: 1005-1012.

5. Ducros A. Reversible cerebral vasoconstriction syndrome. Lancet Neurol. $2012 ; 11$ :906917.

6. Dodick DW, Brown RD Jr, Britton JW, Huston J 3rd. Nonaneurysmal thunderclap headache with diff use, multifocal, segmental, and reversible vasospasm. Cephalalgia 1999; 19: 11823.

7. Miller TR, Shivashankar R, Mossa-Basha M, Gandhi D. Reversible Cerebral Vasoconstriction Syndrome, Part 1: Epidemiology, Pathogenesis, and Clinical Course. AJNR 2015; 36:1392-1399.

8. Ducros A, Fielder U, Porcher R, Boukobza M, Stapf C, Bousser MG. Hemorrhagic manifestations of reversible cerebral vasoconstriction syndrome: frequency, features, and risk factors. Stroke 2010; 41: 2505-2511.

9. Skeik N, Porten BR, Kadkhodayan Y, McDonald W, Lahham F. Postpartum reversible cerebral vasoconstriction syndrome: Review and analysis of the current data. Vasc Med. 2015 ;20: 256-265.

10. Yancy H1, Lee-Iannotti JK, Schwedt TJ, Dodick DW. Reversible cerebral vasoconstriction syndrome. Headache. $2013 ; 53: 570-576$.

11. Fugate JE, Amerisio SF, Ortiz G, Schottlaender LV, Wijdicks EFM, Flemming KD, Rabinstein AA. Variable presentations of postpartum angiopathy. Stroke 2012; 43: 670-676. 
12. Williams TL, Lukovits TG, Harris BT, Harker Rhodes C. A fatal case of postpartum cerebral angiopathy with literature review. Arch Gynecol Obstet 2007; 275: 67-77.

13. Ruzic Y, Tran-Van D, Omarjee A, Boukerrou M, Winer A. Intracerebral haemorrhage and postpartum cerebral angiopathy associated with the administration of sulprostone and norepinephrine. Ann Fr Anesth Reanim 2012; 31: 78-81 .

14. Akhter T, Larsson A, Larsson M, Wikström AK, Naessen T. Artery wall layer dimensions during normal pregnancy: a longitudinal study using noninvasive high-frequency ultrasound. Am J Physiol Heart Circ Physiol 2013; 304: H229-H234.

15. Del Zotto E, Giossi A, Volonghi I, Costa P, Padovani A, Pezzini A. Ischemic Stroke during Pregnancy and Puerperium. Stroke Res Treat 2011 jan 27;2011:6060780. doi: $10.4061 / 2011 / 606780$.

16. Cantu-Brito C, Arauz A, Aburto Y, Barinagarrementeria F, Ruiz-sandoval JL, Baizabalcarvallo JF. Cerebrovascular complications during pregnancy and postpartum: clinical and prognosis observations in 240 Hispanic women. European Journal of Neurology 2011; 18: 819-825.

17. Lim SY, Evangelou N, Jurgens S. Postpartum headache: Diagnostic Considerations. Pract neurol 2014; 14: 92-99.

18. Fugate JE, Wijdicks EF, Parisi JE, et al. Fulminant postpartum cerebral vasoconstriction syndrome. Arch Neurol 2012; 69: 111-17

19. Singhal AB, Taylor Kimberly W, Schaefer PW, Hedley-Whyte E. Case 8-2009: A 36-yearold woman with headache, hypertension, and seizure 2 weeks post partum. N Engl J Med 2009;360:1126-1137.

20. Bartels E. Color-Coded Duplex Ultrasonosgraphy of the cerebral vessels7Atlas and manual. Schattauer . Stuttgart 1999.

21. Felberg RA, Christou I, Demchuk AM, Malkoff M, Alexandrov AV. Screening for Intracranial Stenosis With Transcranial Doppler: The Accuracy of Mean Flow Velocity Thresholds. Journal of Neuroimaging 2002; 12: 1-6.

22. Lindegaard KF, Nornes H, Bakke SJ, Sorteberg W, Nakstad P . cerebral vasospasm diagnosis by means of angiography and blood velocity measurements. Acta Neurochirurgica 1989; 100: 12-24. 
23. Brass L M, Pavlakis S G, DeVivo D, Piomelli S, Mohr J P. Transcranial Doppler measurements of the middle cerebral artery. Effect of hematocrit. Stroke 1988; 19: 1466$\underline{1469 .}$

24. Miller TR (b), Shivashankar R, Mossa-Basha M, Gandhi D. Reversible Cerebral Vasoconstriction Syndrome, Part 2: Diagnostic Work-Up, Imaging Evaluation, and Differential Diagnosis. AJNR 2015; 36: 1580-1588.

25. Albano B, Del Sette M, Roccatagliata L, Gandolfo C, Primavera A.Cortical subarachnoid hemorrhage associated with reversible cerebral vasoconstriction syndrome after elective triplet cesarean delivery. Neurol Sci. 2011 Jun;32(3):497-501.

26. Chen SP, Fuh JL, Chang FC, Lirng JF, Shia BC, Wang SJ. Transcranial color Doppler study for reversible cerebral vasoconstriction syndromes. Ann Neurol 2008;63:751-757.

27. Fugate JE, Rabinstein AA. Posterior reversible encephalopathy syndrome: clinical and radiological manifestations, pathophysiology and outstanding questions.. Lancet Neurol 2015; 14: 914-925.

28. Schwedt TJ, Matharu MS, Dodick D. Thundereclap headache. Lancet Neurol 2006; 5: 621631 